\title{
ANTROPOLOGÍA
}

\section{Vulnerabilidad del VIH/SIDA en los ancianos: una revisión integradora de la literatura}

\author{
Vulnerability of HIV/AIDS in elderly: a review of integrative literature \\ Vulnerabilidade do HIV/AIDS no idoso: uma revisão integrativa de literatura
}

\section{Thais Moraes de Oliveira1 ${ }^{1}$, Carla Lube de Pinho Chibante ${ }^{2}$, Fátima Helena do Espírito Santo $^{3}$, Thayane Dias dos Santos ${ }^{4}$}

${ }^{1}$ Enfermeira. Pós Graduanda em Enfermagem Gerontológica da Universidade Federal Fluminense/UFF. Correo electrónico: thaismoraesdeoliveira@yahoo.com.br

${ }^{2}$ Enfermeira. Doutoranda do Programa Acadêmico em Ciências do Cuidado em Saúde da

Universidade Federal Fluminense/ PACCS-UFF.

Correo electrónico: carla-chibante@hotmail.com

${ }^{3}$ Enfermeira. Prof ${ }^{a}$ Dr $^{a}$ do Departamento de Enfermagem Médico-Cirúrgica da Escola de Enfermagem Aurora de Afonso Costa/UFF. Coordenadora da Pós Graduação em Enfermagem Gerontológica/UFF. Correo electrónico: fatahelen@hotmail.com

${ }^{4}$ Enfermeira. Mestre em Ciências do Cuidado em Saúde da Universidade Federal Fluminense/UFF. Correo electrónico: thayanedsantos@hotmail.com

Cómo citar este artículo en edición digital: Oliveira, T.M., Pinho Chibante, C.L., Espírito Santo, F.H. y dos Santos, T.D. (2020). Vulnerabilidad del VIH/SIDA en los ancianos: una revisión integradora de la literatura. Cultura de los Cuidados (Edición digital), 24 (56) Recuperado de http://dx.doi.org/10.14198/cuid.2020.56.10

Correspondencia: Rua Silveira Martins, 164/ Apt ${ }^{\circ} 905$, Catete, CEP: 22221000. Rio de Janeiro (RJ). Tel: 21983381243.

Correo electrónico de contacto: carla-chibante@ig.com.br

Recibido:14/11/2029 Aceptado:13/12/2020

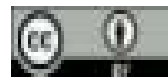

ABSTRACT

Objective: To characterize the scientific production on the HIV/AIDS vulnerability in the elderly. Method: Integrative revision, from the guiding question how is HIV/AIDS vulnerability being approached in Brazilian literature? The search for scientific production was conducted on MEDLINE, BDENF and LILACS data base, employing the HIV, aged and health vulnerability descriptors. Results: 19 articles divided into two categories: vulnerability and HIV in elderly people were found; And the diagnosis and intervention in elderly with HIV. Conclusion: The stigma of the elderly population, prejudice and belief that aging is related to the loss of sexual desire causes an 
increase in the number of elderly people with HIV/AIDS. It is necessary for the studies to address the issues of the social representation of HIV to the elderly, in addition to specific interventions to this age group to improve the quality of life of this population.

Keywords: HIV, aged, health vulnerability.

\section{RESUMEN}

Objetivo: caracterizar la producción científica sobre la vulnerabilidad del VIH/SIDA en los ancianos. Método: revisión Integrativa, de la pregunta orientadora ¿cómo se está abordando la vulnerabilidad del VIH/SIDA en la literatura brasileña? La búsqueda de producción científica se llevó a cabo en MEDLINE, BDENF y LILACS data base, empleando el descriptores VIH, anciano y vulnerabilidade em salud. Resultados: 19 artículos divididos en dos categorías: se encontró la vulnerabilidad y el VIH en personas de edad avanzada; Y el diagnóstico y la intervención en ancianos con VIH. Conclusión: el estigma de la población de edad avanzada, el prejuicio y la creencia de que el envejecimiento está relacionado con la pérdida de deseo sexual causa un aumento en el número de personas mayores con VIH/SIDA. Es necesario que los estudios se enfrenten a las cuestiones de la representación social del VIH a los ancianos, además de las intervenciones específicas a este grupo de edad para mejorar la calidad de vida de esta población.

Palabras clave: VIH, anciano, vulnerabilidade em salud.

\section{RESUMO}

Objetivo: caracterizar a produção científica sobre a vulnerabilidade do HIV/AIDS no idoso. Método: revisão integrativa, a partir da questão norteadora como a vulnerabilidade do HIV/AIDS vem sendo abordada na literatura brasileira?. Realizou-se a busca da produção científica na base de dados MEDLINE, BDENF e LILACS, empregando os descritores HIV, Idoso e Vulnerabilidade em Saúde. Resultados: foram encontrados 19 artigos divididos em duas categorias: a vulnerabilidade e o HIV em idosos; e o diagnóstico e intervenção em idosos com HIV. Conclusão: O estigma da população idosa, o preconceito e a crença que o envelhecimento está relacionado à perda do desejo sexual faz com que tenha um aumento no número de idosos com HIV/AIDS. É preciso que os estudos abordem as questões da representação social do HIV traz aos idosos, além de intervenções específicas a esta faixa etária para melhorar a qualidade de vida desta população.

Palavras chave: HIV, idoso, vulnerabilidade em saúde.

\section{INTRODUÇÃO}

A justificativa para a realização desta pesquisa aconteceu por meio da atuação como enfermeira em uma instituição privada do Rio de Janeiro, em que foi observado que a maioria dos pacientes era de pessoas idosas e estavam com o Vírus da Imunodeficiência Humana (HIV).

Nesse caso, pode-se verificar que esta faixa etária, atualmente, encontra-se bastante vulnerável a esta doença, pois há aspectos intrínsecos tanto da sociedade quanto do próprio idoso que colaboram para o aumento do número de casos.

No Brasil, há mais de 20 milhões de pessoas com mais de 60, o que representa 10\% da população. Em 2050, estima-se que esse número cresça para $30 \%$. Este fenômeno ocorre por causa do aumento da expectativa de vida e queda na mortalidade da população brasileira. Ademais, a urbanização, bem como o avanço da tecnologia na saúde, diminuiu o índice de mortalidade e morbidade nesta população (Dornelas et al, 2015).

Com sua evolução, percebeu-se que a prevalência de casos em indivíduos idosos 
Revista científica de la Asociación de Historia y Antropología de los Cuidados (Universidad de Alicante)

aumentou. Na década de 80 até meados de 2013, o Ministério da Saúde constatou mais de 686 mil casos de AIDS, dentre os quais 445 mil eram do sexo masculino e 241 eram do sexo feminino (Santana et al, 2015).

No período de 1996 a 2006, entre os homens idosos com HIV, o índice por 100 mil habitantes passou de 5,9 para 8,8. Entre as mulheres, houve um crescimento de 1,7 para 5,1. Esse crescimento pode estar associado ao aumento do envelhecimento populacional brasileiro e, consequentemente, com a melhora da qualidade de vida, o que prolonga a vida sexual e à existência do tabu de se falar sobre sexualidade nessa faixa etária (Lima e Freitas, 2013).

Os primeiros casos de HIV no Brasil e no mundo começaram a partir da década de 80 e estavam relacionados aos homossexuais, profissionais do sexo e usuários de drogas. Em seu início, os idosos não faziam parte dos grupos vulneráveis e as campanhas de prevenção eram reduzidas com relação a esse tipo de grupo. Fato que explica o porquê desta faixa etária ter dificuldades em aderir os métodos de prevenção da doença (Affeldt, Silveira e Barcelos, 2015).

Nesse contexto, com a epidemia do HIV/AIDS, não há mais os chamados “grupos de risco”. Isto ocorre porque, no decorrer dos anos, houve uma mudança no comportamento sexual, na juventude, na feminilidade e no envelhecimento da população brasileira. Além disso, a dificuldade de detecção do estado sorológico também é uma das causas da vulnerabilidade, pois muitos idosos pensam que esta doença está restrita somente as pessoas jovens, homossexuais e profissionais do sexo (Quadros et al, 2016).

Além disso, a prática sexual não segura, sem o uso de preservativos, também é considerada outro problema com relação ao vírus. Ademais, a sociedade e as instituições de saúde, até pouco tempo, desconsideravam a ideia de contágio do HIV nesta faixa etária (Dornelas et al, 2015).

Diante desse fato, é imprescindível que haja um conhecimento mais amplo do HIV/AIDS na população idosa, a fim de remover obstáculos que impedem que o mesmo utilize práticas seguras de prevenção. O número de pessoas com HIV, constitui um evento global, dinâmico e oscilante, pois a forma de ocorrência em diversos locais do mundo depende de outros fatores, como por exemplo, o próprio comportamento do indivíduo e das pessoas que o cercam (Lima e Freitas, 2013).

Além disso, a vida sexual ativa desta faixa etária é constantemente influenciada pela indústria farmacêutica que, por sua vez, disponibiliza medicações para a disfunção erétil. Simultaneamente, há uma desmistificação do sexo, o que pode ocasionar uma maior vulnerabilidade com relação ao HIV (Bittencourt et al, 2015).

Nesse contexto, quando comparado aos jovens, os idosos são diagnosticados em um estágio avançado desta patologia. Diante 
Cultura de los Cuidados

deste fenômeno, há alguns fatores que contribuem para este processo, que são: a escassez de profissionais de saúde para suspeitar desta doença, ausência de exames de rotina, desconhecimento, pensamento de que não vai adquirir o vírus por causa da idade e entre outros (Mpondo, 2016).

Uma vez que a AIDS é considerada uma doença crônica e degenerativa, esta pode ser prevenida por meio de intervenções e medidas eficazes. O tratamento, nesse caso, permite que o indivíduo tenha uma maior sobrevida, contudo, sua acessibilidade e aceitabilidade estão entre os maiores impasses no controle deste vírus (Okuno et al, 2014). Por isso, sua vulnerabilidade envolve alguns fatores, que são o individual, social e o institucional, que apresentam visões distintas quanto se trata da prevenção. A primeira expõe a qualidade e o alcance da informação recebida. A segunda, descreve as condições de bem-estar social e, a última, fala sobre a preocupação do governo sobre este problema (Bezerra et al, 2015).

Dessa forma, constitui-se como objeto deste estudo a vulnerabilidade do HIV/AIDS no idoso. Delimitou-se como questão norteadora: Como a vulnerabilidade do HIV/AIDS vem sendo abordada na literatura brasileira? $\mathrm{O}$ objetivo foi caracterizar a produção científica sobre a vulnerabilidade do HIV/AIDS no idoso.

\section{TRAJETÓRIA METODOLÓGICA}

Este artigo consiste em uma revisão integrativa da literatura, com abordagem exploratória e método descritivo.
A revisão integrativa analisa as pesquisas relevantes que dão a base para a tomada de decisão e aperfeiçoamento da prática assistencial, o que possibilita um compêndio do conhecimento de um assunto além de revelar lacunas do conhecimento que necessitam ser completadas com a realização de novos trabalhos científicos (Mendes, Silveira e Galvão, 2008).

Os passos para estudos de revisão integrativa são: Primeira etapa: identificação do tema e seleção da hipótese ou questão de pesquisa para a elaboração da revisão integrativa; Segunda etapa: estabelecimento de critérios para inclusão e exclusão de estudos/ amostragem ou busca na literatura; Terceira etapa: definição das informações a serem extraídas dos estudos selecionados/ categorização dos estudos; Quarta etapa: avaliação dos estudos incluídos na revisão integrativa; Quinta etapa: interpretação dos resultados e Sexta etapa: apresentação da revisão/síntese do conhecimento (Mendes, Silveira e Galvão, 2008).

A busca na literatura foi realizada nas bases de dados: Medical Literature Analysis and Retrieval System Online (MEDLINE); Base de Dados da Enfermagem (BDENF); e Literatura LatinoAmericana e do Caribe em Ciências da Saúde (LILACS), por meio da Biblioteca Virtual de Saúde (BVS).

O período de coleta de dados ocorreu de agosto a novembro de 2016. Os critérios para a inclusão das pesquisas foram: artigos indexados nas bases de dados, no idioma português e inglês, com o recorte 
Revista científica de la Asociación de Historia y Antropología de los Cuidados (Universidad de Alicante)

temporal de 2011 a 2015, utilizando os descritores "HIV", "Idoso" e “Vulnerabilidade em saúde”. Os critérios de exclusão foram: estudos que não possuíam texto completo disponível gratuitamente; repetidos nas bases de dados e que não apresentavam relação com a temática. Ao realizar o somatório de todos os artigos que atenderam aos critérios de inclusão préestabelecidos, totalizou-se 29 artigos, que foram seguidamente submetidos à leitura e análise de seus títulos e resumos. Neste contexto, com a aplicabilidade dos critérios de exclusão: 5 (17\%) foram excluídos por não estarem relacionados à temática; 3 $(10,34 \%)$ por se repetirem nas bases de dados e 2 (6,9\%) por não apresentarem disponibilidade do seu conteúdo na íntegra, gratuitamente. Totalizando um quantitativo de 10 (34, 4\%) artigos excluídos.

Após a seleção dos 19 artigos, os mesmos foram analisados de forma crítica, expondo os resultados encontrados, dando origem à duas categorias: a vulnerabilidade e o HIV em idosos; e o diagnóstico e intervenção em idosos com HIV. Posteriormente, discutidos de modo a comparar os principais achados com os demais estudos da literatura.

\section{RESULTADOS}

Feitas as associações dos descritores nas bases de dados, foram encontrados 29 artigos e 19 selecionados para compor este estudo, conforme apresentado no quadro 1. De acordo com a base de dados, 10 (52,63\%) na LILACS, 5 (26,3\%) na Medline e 4
(21,05\%) na BDENF. Com relação ao ano, 5 (26,3\%) em 2015, 4 (21,05\%) em 2011, 2012 e 2013, respectivamente, e 2 (10,5\%) em 2014. Quanto à formação dos autores, 14 $(73,31 \%)$ foram realizados por enfermeiros e 5 (26,3\%) por médicos. Conforme o tipo de estudo, 9 (47,3\%) eram descritivos, 4 (21,05\%) qualitativos, 2 (10,5\%) retrospectivos, 1 (5,26\%) temporal, revisão integrativa, não sistemática e transversal, respectivamente. Com relação às regiões, cujos estudos foram realizados, 6 (31,5\%) em São Paulo, 4 (21,05\%) no estado da Paraíba, 3 (15,7\%) no Rio de Janeiro, 2 (10,5\%) em Minas Gerais, e 1 (5,26\%) nos estados do Rio Grande do Sul, Piauí, Mato Grosso do Sul e Rio Grande do Norte, respectivamente.

No que diz respeito às revistas onde os artigos foram publicados, 4 (21,5\%) na Revista Brasileira de Geriatria e Gerontologia, 2 (10,5\%) na Revista Brasileira de Enfermagem, DST-Jornal Brasileiro de Doenças Sexualmente Transmissíveis, Revista Mineira de Enfermagem e Caderno de Saúde Pública, respectivamente, e 1 (5,26\%) na Revista Gaúcha de Enfermagem, Cuidarte Enfermagem, Revista de Enfermagem USP, Revista Latino Americana de Enfermagem, Revista de Enfermagem UERJ, Clinics e Revista Eletrônica de Enfermagem. 
Quadro 1 - Artigos selecionados quanto ao ano de publicação, periódico, título e principais resultados, 2016.

\begin{tabular}{|c|c|c|c|}
\hline $\begin{array}{c}\text { Ano e base de } \\
\text { dados }\end{array}$ & Periódico (Revista) & Título do Artigo & Principais resultados \\
\hline 2015/ Lilacs & $\begin{array}{l}\text { Revista Brasileira de } \\
\text { Enfermagem- REBEN }\end{array}$ & $\begin{array}{l}\text { Concepções de idosos sobre } \\
\text { vulnerabilidade ao HIV/Aids } \\
\text { para construção de } \\
\text { diagnósticos de enfermagem }\end{array}$ & $\begin{array}{l}\text { Identificaram-se os diagnósticos } \\
\text { conhecimento sobre comportamento } \\
\text { sexual adequado, capacidade para } \\
\text { proteção parcial, medo da morte e } \\
\text { desesperança. }\end{array}$ \\
\hline 2015/Bdenf & $\begin{array}{l}\text { Caderno de Saúde } \\
\text { Pública }\end{array}$ & $\begin{array}{llr}\text { Dinâmica } & \text { espacial } & \text { da } \\
\text { incidência } & \text { da AIDS } & \text { em } \\
\text { idosos no Rio de Janeiro, } \\
\text { Brasil, 1997-2011 }\end{array}$ & $\begin{array}{l}\text { Os índices de AIDS em idosos têm } \\
\text { estabilizado nas últimas décadas. } \\
\text { Maior atenção deve ser dada ao gru- } \\
\text { po idoso para evitar a progressão da } \\
\text { doença na população. }\end{array}$ \\
\hline 2015/Medline & $\begin{array}{c}\text { Revista Brasileira de } \\
\text { Geriatria e } \\
\text { Gerontologia }\end{array}$ & $\begin{array}{l}\text { Impacto psicossocial } \\
\text { diagnóstico de HIV/aids em } \\
\text { idosos atendidos em um } \\
\text { serviço público de saúde }\end{array}$ & $\begin{array}{l}\text { Diagnóstico de HIV/aids implica } \\
\text { uma reorganização na maneira como } \\
\text { se relacionam com pessoas; no } \\
\text { autocuidado; nas práticas sexuais; na } \\
\text { realização de atividades cotidianas e } \\
\text { nas possibilidades de manterem sua } \\
\text { participação em grupos sociais. }\end{array}$ \\
\hline 2015/ Medline & $\begin{array}{l}\text { Revista Gaúcha de } \\
\text { Enfermagem }\end{array}$ & $\begin{array}{l}\text { Práticas preventivas de } \\
\text { idosos e a vulnerabilidade ao } \\
\text { HIV }\end{array}$ & $\begin{array}{l}\text { Os idosos apontaram grupos } \\
\text { populacionais mais vulneráveis ao } \\
\text { HIV e não se reconhecem como tal. }\end{array}$ \\
\hline 2015/Lilacs & Cuidarte Enfermagem & $\begin{array}{lr}\text { Sexualidade } & \mathrm{e} \\
\text { comportamento dos } & \text { idosos } \\
\text { vulneráveis a doenças } \\
\text { sexualmente transmissíveis. }\end{array}$ & $\begin{array}{l}\text { Verificou-se um aumento dos casos } \\
\text { de doenças pelo HIV no município } \\
\text { de Catanduva-SP, ratificando os } \\
\text { resultados dos questionários, os } \\
\text { quais demonstraram } \\
\text { vulnerabilidade dos idosos } \\
\text { sexualmente ativos pela prática } \\
\text { desprotegida }\end{array}$ \\
\hline 2014/Bdenf & $\begin{array}{l}\text { Revista Escola de } \\
\text { Enfermagem USP }\end{array}$ & $\begin{array}{l}\text { O diagnóstico tardio e as } \\
\text { vulnerabilidades dos idosos } \\
\text { vivendo com HIV/aids }\end{array}$ & $\begin{array}{l}\text { Do processo de análise, segundo a } \\
\text { Análise de Conteúdo, emergiram } \\
\text { quatro categorias: descobrindo-se } \\
\text { tardiamente com HIV na contramão } \\
\text { do serviço de saúde, percebendo-se } \\
\text { assexuado pelos profissionais da } \\
\text { saúde }\end{array}$ \\
\hline 2014/Lilacs & $\begin{array}{l}\text { Revista Latino } \\
\text { Americana de } \\
\text { Enfermagem }\end{array}$ & $\begin{array}{l}\text { Vulnerabilidade de mulheres } \\
\text { vivendo com HIV/Aids }\end{array}$ & $\begin{array}{l}\text { Predominaram mulheres brancas, } \\
\text { entre } 30 \text { e } 49 \text { anos de idade, com } \\
\text { companheiro, baixo nível escolar, } \\
\text { múltiplos parceiros sexuais durante a } \\
\text { vida e prática de sexo inseguro }\end{array}$ \\
\hline 2013/ Lilacs & $\begin{array}{c}\text { Revista Brasileira de } \\
\text { Geriatria e } \\
\text { Gerontologia }\end{array}$ & $\begin{array}{l}\text { Oficinas educativas sobre } \\
\text { HIV/Aids: uma proposta de } \\
\text { intervenção para idosos }\end{array}$ & $\begin{array}{l}\text { O domínio "vulnerabilidade" } \\
\text { indicou 34,93\% na questão da aids } \\
\text { relacionada a grupos específicos }\end{array}$ \\
\hline 2013/ Medline & $\begin{array}{c}\text { Caderno de Saúde } \\
\text { Pública }\end{array}$ & $\begin{array}{l}\text { Caracterização } \\
\text { epidemiológica dos casos de } \\
\text { AIDS em pessoas com } 60 \\
\text { anos ou mais, Pernambuco, } \\
\text { Brasil, } 1998 \text { a } 2008\end{array}$ & $\begin{array}{l}\text { Em } 1998 \text { a taxa de incidência de } \\
\text { AIDS, na população de estudo, era } \\
\text { de } 1,6 \text { caso } / 100 \text { mil habitantes, } \\
\text { passando para } 4,8 \text { casos } / 100 \text { mil } \\
\text { habitantes em } 2008 \text {, caracterizando } \\
\text { um aumento de } 200 \% \text {. }\end{array}$ \\
\hline
\end{tabular}




\begin{tabular}{|c|c|c|c|}
\hline 2013/ Medline & $\begin{array}{l}\text { DST - Jornal Brasileiro de } \\
\text { Doenças Sexualmente } \\
\text { Transmissíveis }\end{array}$ & $\begin{array}{l}\text { A influência do uso da camisinha } \\
\text { por idosos na vulnerabilidade ao } \\
\text { HIV: uma revisão sistemática } \\
\text { com meta análise }\end{array}$ & $\begin{array}{l}\text { Foram selecionados } 38 \text { artigos e, após } \\
\text { avaliação e aplicação da escala } \\
\text { Physiotherapy Evidence Database } \\
\text { (PEDro), foram eleitos } 6 \text { deles para } \\
\text { compor a amostra do estudo. Mediante } \\
\text { análise estatística, obteve-se uma medida } \\
\text { sumário }\end{array}$ \\
\hline 2013/Bdenf & $\begin{array}{c}\text { Revista de Enfermagem } \\
\text { UERJ }\end{array}$ & $\begin{array}{l}\text { Percepção de mulheres casadas } \\
\text { sobre o risco de infecção pelo } \\
\text { HIV e o comportamento } \\
\text { preventivo }\end{array}$ & $\begin{array}{l}\text { A partir da análise dos dados emergiram } \\
\text { três categorias: transmissão do } \\
\text { HIV/AIDS, vulnerabilidade ao HIV/ } \\
\text { AIDS e prevenção do HIV/AIDS. }\end{array}$ \\
\hline 2012/ Lilacs & $\begin{array}{l}\text { DST - Jornal Brasileiro de } \\
\text { Doenças Sexualmente } \\
\text { Transmissíveis }\end{array}$ & $\begin{array}{l}\text { Vulnerabilidade dos Idosos } \\
\text { frente ao HIV/Aids: Tendências } \\
\text { da Produção Científica Atual no } \\
\text { Brasil }\end{array}$ & $\begin{array}{l}\text { Dentre os mais encontrados nas leituras } \\
\text { realizadas, estão: existência de tabus } \\
\text { sobre a sexualidade na velhice e } \\
\text { conhecimento escasso dos idosos sobre a } \\
\text { infecção pelo HIV. }\end{array}$ \\
\hline 2012/Lilacs & $\begin{array}{l}\text { Revista Brasileira de } \\
\text { Enfermagem- REBEN }\end{array}$ & $\begin{array}{l}\text { Comportamentos em saúde de } \\
\text { uma população portadora do } \\
\text { HIV/AIDS }\end{array}$ & $\begin{array}{l}\text { Relataram dificuldade para utilizar o } \\
\text { preservativo (22,2\% dos homens) pelo } \\
\text { comprometimento da ereção; 97,2\% } \\
\text { referiram não usar drogas para } \\
\text { estimulação sexual; 90,8\% afirmaram não } \\
\text { ter usado proteção nas relações antes de } \\
\text { se saberem infectados }\end{array}$ \\
\hline 2012/Lilacs & $\begin{array}{l}\text { Revista Brasileira de } \\
\text { Geriatria e Gerontologia }\end{array}$ & $\begin{array}{l}\text { Análise da clientela idosa } \\
\text { portadora de HIV atendida em } \\
\text { um centro ambulatorial em } \\
\text { Montes Claros, Minas Gerais }\end{array}$ & $\begin{array}{l}6,2 \% \text { possuíam parceria única. } 53,9 \% \\
\text { usavam condom durante relação sexual; } \\
100 \text { se contaminaram por via sexual; } \\
53,9 \% \text { adquiriram a infecção de } \\
\text { profissionais do sexo; } 76,9 \% \text { faziam uso } \\
\text { de antirretrovirais; }\end{array}$ \\
\hline 2012/Medline & Clinics & $\begin{array}{l}\text { Vulnerabilidade da AIDS em } \\
\text { idoso em um centro urbano no } \\
\text { Brasil }\end{array}$ & $\begin{array}{l}\text { A maioria dos idosos na pesquisa era } \\
\text { independente, na faixa etária dos } 60 \text { aos } \\
69 \text {, que tem conhecimento sobre a AIDS } \\
\text { e estavam cientes que eram vulneráveis à } \\
\text { doença. }\end{array}$ \\
\hline 2011/Lilacs & $\begin{array}{l}\text { Revista Mineira de } \\
\text { Enfermagem }\end{array}$ & $\begin{array}{l}\text { Adesão dos portadores do } \\
\text { HIV/AIDS ao tratamento: fatores } \\
\text { intervenientes }\end{array}$ & $\begin{array}{l}\text { Os resultados também evidenciaram que } \\
\text { a maioria (60\%) faz uso regular dos } \\
\text { antirretrovirais, mesmo apresentando } \\
\text { efeitos colaterais. No que se refere às } \\
\text { medidas não medicamentosas, a maioria } \\
\text { (87,5\%) afirma que não realiza atividades } \\
\text { físicas }\end{array}$ \\
\hline 2011/ Bdenf & $\begin{array}{l}\text { Revista Eletrônica de } \\
\text { Enfermagem }\end{array}$ & $\begin{array}{l}\text { Perfil clínico e epidemiológico da } \\
\text { infeç̧ão pelo HIV/aids em idosos }\end{array}$ & $\begin{array}{l}\text { Dos } 208 \text { prontuários analisados, } 45,7 \% \\
\text { eram de mulheres e } 54,3 \% \text { de homens, } \\
\text { com idade média de } 60,6 \text { anos. } \\
\text { Categoria de exposição sexual foi a } \\
\text { predominante e quanto ao CD4, } 68,2 \% \\
\text { apresentaram contagem menor que } 200 \\
\text { cel/mm3. A mortalidade foi elevada } \\
(44,7 \%)\end{array}$ \\
\hline 2011/Bdenf & $\begin{array}{c}\text { Revista Brasileira de } \\
\text { Geriatria e Gerontologia }\end{array}$ & $\begin{array}{l}\text { Vulnerabilidade das idosas ao } \\
\text { HIV/AIDS: despertar das } \\
\text { políticas públicas e profissionais } \\
\text { de saúde no contexto da atenção } \\
\text { integral: revisão de literatura }\end{array}$ & $\begin{array}{l}\text { Abordagem integral contribui para o } \\
\text { entendimento do processo de } \\
\text { adoecimento, especialmente naquele } \\
\text { indivíduo com o vírus HIV, que vivencia } \\
\text { preconceitos, estigmas e discriminação, } \\
\text { desafiando as estratégias de prevenção de } \\
\text { novas infecções e ações de assistência à } \\
\text { saúde mais apropriadas. }\end{array}$ \\
\hline 2011/Lilacs & $\begin{array}{l}\text { Revista Mineira de } \\
\text { Enfermagem }\end{array}$ & $\begin{array}{l}\text { Vulnerabilidade em face das } \\
\text { infecções sexualmente } \\
\text { transmissíveis e HIV/AIDS nos } \\
\text { roteiros sexuais de mulheres com } \\
\text { transtornos mentais }\end{array}$ & $\begin{array}{l}\text { A vulnerabilidade mostrou-se agravada } \\
\text { pelo contexto de exclusão social em que } \\
\text { vive esse grupo populacional. }\end{array}$ \\
\hline 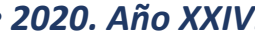 & & & \\
\hline
\end{tabular}


Após a leitura minuciosa de cada estudo, os mesmos foram organizados, de modo a favorecer a exposição e discussão dos resultados, em 2 categorias: a questão da vulnerabilidade e o HIV em idosos, em que se concentraram dez (10) artigos; e a questão do diagnóstico e intervenção em idosos com HIV, contemplando nove (9) artigos.

\section{A VULNERABILIDADE E O HIV EM IDOSOS}

A sexualidade faz parte da essência humana em qualquer idade, mas, quando ela está associada à população idosa, apresentase rodeada de mitos e crenças. Nesse contexto, é muito comum a sociedade atual e os profissionais de enfermagem pensarem que o processo de envelhecimento está associado a perda do desejo sexual (Alencas e Ciosak, 2014).

Diante disso, a possibilidade de uma pessoa idosa ser infectada pelo vírus HIV parece não ter visibilidade na sociedade atual. É incômodo admitir ou até mesmo conversar sobre este assunto, pois ainda é tabu e as pessoas preferem estigmatizar e discriminar idosos com relação a sua vida sexual (Santos e Assis, 2011).

Observa-se, também, que esta faixa etária não tem a informação adequada vindo dos profissionais de saúde, principalmente dos enfermeiros, sobre o vírus. Nesse caso, muitos buscam meios de comunicação, tais como televisão, rádio e jornais, como suas principais referências. Com isso, deixam de detectar precocemente o vírus, ao prestar uma abordagem inadequada da sexualidade dessas pessoas e o exame imediato (sorologia para o HIV) após a observação e o relato dos primeiros sinais e sintomas (Garcia et al, 2012).

Aliado a isso, o aumento da expectativa de vida dos idosos associado a modernização da saúde, que neste caso incluem a reposição hormonal e as medicações para melhorar o desempenho sexual, abre lacunas para que os mesmos tenham a oportunidade de redescobrir experiências no que diz respeito à sua sexualidade (Alencas e Ciosak, 2014).

Além disso, as questões de gênero, ou seja, o comportamento sexual previamente definido e socialmente exigido de homens e mulheres é um dos problemas que agravam a prevenção do HIV/AIDS, o que favorece uma vulnerabilidade individual no contexto das relações de gênero, por se tratarem de construções sociais com repercussões na saúde das pessoas envolvidas (Bezerra et al, 2015).

Nesse contexto, a população do sexo masculino tem medo de perder a ereção e as mulheres, por não poderem engravidar, acreditam que não precisam mais se prevenir, aumentando as chances referentes às Doenças Sexualmente Transmissíveis (DSTs). Ressalta-se ainda que muitos não foram instruídos a usarem os preservativos na juventude, o que desencadeia a resistência a esse tipo de proteção (Burigo et al, 2015).

Corroborando com o autor acima, há outros motivos que levam os dois gêneros a não usarem preservativo, que são: os homens 
Revista científica de la Asociación de Historia y Antropología de los Cuidados (Universidad de Alicante)

acham que o cuidado é necessário somente nas relações com as profissionais do sexo. Já as mulheres não sentem necessidade de exigir o preservativo, porque já perderam a capacidade de engravidar e consideram que não precisam mais de prevenção (Santos e Assis, 2011).

Associada a falta do uso do preservativo, há outros comportamentos de risco mais frequentes, que são: parceiro portador de HIV, promiscuidade sexual, baixo grau de instrução e relações extraconjugais, evidenciando que a categoria de exposição para essa faixa etária é a sexual e por meio de relação heterossexual (Ultramari, 2011).

A via heterossexual é a categoria de maior proporção e isso reforça que a prática sexual, sem proteção, constitui a mais importante via de transmissão também entre os idosos, mostrando a necessidade de abordagens para o sexo seguro também para esta faixa etária. Outro aspecto importante é que, para as mulheres mais velhas, o sexo ainda é tabu e para os homens, têm menos barreiras, independentemente do status de relacionamento (Silva, Vasconcelos e Ribeiro, 2013; Driemeier et al, 2012).

Com isso, os idosos representam uma população vulnerável ao HIV/AIDS devido a estes fatores comportamentais. E isso acontece por causa das métropoles brasileiras, que desempenham um papel importante na propagação desta doença, já que as mesmas são centros de comércio e interação social (Rodrigues et al, 2015).
Diante disso, os idosos são considerados uma faixa etária vulnerável, uma vez que que há uma negligência por parte da sociedade e também dos profissionais de enfermagem quanto ao HIV/AIDS. Diante deste fato, o próximo tópico discutirá as causas do diagnóstico tardio e os tipos de intervenções que possam ser colocadas em prática para aumentar a prevenção no que diz respeito a essa doença.

\section{O DIAGNÓSTICO E INTERVENÇÃO} EM IDOSOS COM HIV

A abordagem da sexualidade na terceira idade, como a vulnerabilidade do HIV são constituídos por meio de um panorama de saúde que gera desafio aos profissionais durante a assistência ao idoso (Bittencourt et al, 2015).

No processo de envelhecimento, o trato genital das mulheres sofre modificações fisiológicas, sendo que as paredes vaginais ficam mais finas e a lubrificação diminui, tornando o meio propício para o aparecimento de lesões durante as relações sexuais, o que aumentaria o risco de serem contaminadas pelo HIV. Salienta-se, nesse caso, a importância do uso do preservativo feminino visando a diminuição da desigualdade de gênero, por permitir que as mulheres pratiquem o autocuidado no que diz respeito a sua vida sexual, independente da escolha do parceiro em relação à prevenção (Lazarotto et al, 2013). 
Para que isso ocorra, há uma necessidade de que as políticas públicas demonstrem essa necessidade do uso do preservativo para a população idosa, objetivando a diminuição da desmistificação da ideia de que a camisinha é usada apenas como método contraceptivo, salientando que a mesma pode prevenir a infecção pelo HIV. Destaca-se também a importância de sensibilizar quanto ao problema, visando a diminuição da resistência ao uso e redução de riscos (Lazarotto et al, 2013).

Outro aspecto relevante é que essas políticas públicas devem ser baseadas na desconstrução de imagens estereotipadas deste tipo de patologia na sociedade, fatores específicos dessa idade, como a dificuldade de mudança de hábitos e inserção de novas formas de lidar com a sexualidade, além de outros importantes, como valores culturais (Lima e Freitas, 2013).

Nesse contexto, as estratégias da enfermagem, diante desse público, são essenciais, pois esta categoria executa o papel de educador, cuja prática se baseia na conscientização e sensibilização dos pacientes sobre a importância da realização dos exames e também da prevenção do HIV (Almeida et al, 2011).

A educação em saúde constitui uma intervenção que visa promover a qualidade de vida da população, família e comunidades mediante a articulação de conhecimentos técnicos e populares, de recursos institucionais e comunitários, de iniciativas públicas e privadas, superando a conceituação biomédica de assistência à saúde e ampliando diversos determinantes do processo saúde-enfermidade-cuidado (Figueiredo et al, 2013).

Dentro desse tipo de processo educativo, a consulta de enfermagem é importante para essa clientela, pois durante a consulta, pode-se abordar o paciente e o familiar, sempre oferecendo orientações sobre a doença e o tratamento correto com o antirretroviral, bem como escutar os medos, queixas e anseios que possam surgir (Almeida et al, 2011).

Os diagnósticos de enfermagem também são outras intervenções que buscam o levantamento de problemas comuns e necessidade de saúde desta faixa etária. Após a identificação dos diagnósticos, é possível realizar uma avaliação de necessidades de saúde e fatores que desencadeiam as vulnerabilidades do idoso ao HIV/AIDS (Bittencourt et al, 2015).

Além disso, o enfermeiro tem como ação distribuir insumos de proteção e informar sobre o uso correto desses preservativos, bem como informar a população sobre os riscos adquiridos do HIV/AIDS na relação sexual desprotegida (Paz et al, 2013).

Ressalta-se ainda que a importância do uso de preservativos deve ser sempre enfatizada, mesmo quando ambos os parceiros estiverem contaminados pelo HIV. Nesse aspecto, o não uso pode propiciar a infecção por outras DSTs, dificultando o controle da carga viral, o que pode aumentar o risco de contaminação por vírus resistentes aos antirretrovirais (Souza et al, 2012). 
Revista científica de la Asociación de Historia y Antropología de los Cuidados (Universidad de Alicante)

Com relação ao diagnóstico do HIV,

o medo é a principal reação da terceira idade quando se depara com o exame. Além disso, o medo da morte, o medo da incapacidade, mas, especialmente, o medo de que esse diagnóstico, considerado constrangedor, fosse revelado aos familiares, amigos e a outras pessoas do convívio social, desencadeando constrangimentos ao idoso, rejeição, discriminação e afastamento das pessoas (Duarte, Parada e Souza, 2014).

Diante deste cenário, os profissionais devem sempre enfatizar que os testes sorológicos são baseados na identificação dos anticorpos ou antígenos do HIV presentes ou não na amostra do paciente. Em pessoas adultas e idosas, esses anticorpos aparecem no sangue dos indivíduos infectados, em média de quatro a 12 semanas após a infecção (Silva et al, 2015).

Nesse sentido, o estigma que o HIV causa à população geral prejudica a adesão dos idosos ao teste de HIV, pois o desconhecimento das informações é o principal fator que estimula esse tipo de atitude. Portanto, o profissional de enfermagem deve levar em consideração o estilo de vida do idoso, seus medos, suas queixas, o nível de informação que ele detém e também o tipo de relação que ele desenvolve com sua família e amigos para que haja uma ação conjunta que ajude a prevenir ou a lidar com esse tipo de doença caso este idoso seja infectado pelo vírus.
CONCLUSÃO

O estigma da população idosa, o preconceito e a crença que o envelhecimento está relacionado à perda do desejo sexual faz com que se tenha um aumento no número de idosos com HIV/Aids. É necessário que os estudos abordem as questões da representação social do HIV traz aos idosos, além de intervenções específicas a esta faixa etária para melhorar a qualidade de vida desta população.

A principal causa que propicia a proliferação do vírus HIV, no idoso, é o estigma que está envolto nessa faixa etária. Nesse contexto, a sociedade e os profissionais de enfermagem desconsideram que esta população possa vir a ter desejo sexual. Diante dessa constatação, a falta de informação contribui para o preconceito, pois existe a crença que o processo de envelhecimento está relacionado a perda do desejo sexual, prejudicando a abordagem dos profissionais da saúde na prevenção do idoso ao HIV.

Quanto ao diagnóstico, o medo causado pelo preconceito da família e até mesmo da sociedade inibem esta faixa etária de fazer o teste de HIV. Nesse caso, para que haja uma intervenção adequada, deve levar em consideração que a educação em saúde constitui o melhor método na abordagem dessa população. Um dos benefícios da educação em saúde é o aumento da qualidade de vida por meio de informações concisas e acessíveis a esta população. 
Sendo assim, outras pesquisas deverão ser realizadas para resolver as possíveis falhas no que tange às representações sociais que o HIV traz a esses idosos e também aos profissionais de enfermagem, já que isso desestimula a prevenção e a busca pelo tratamento.

Logo, os próximos artigos científicos poderiam abordar outros tipos de intervenções que fossem direcionadas a população idosa, uma vez que há uma escassez de pesquisas que descrevem a atuação do profissional de enfermagem em idosos com HIV.

\section{REFERÊNCIAS}

Affeldt, A.B., Silveira, M.F., Barcelos, R.S. (2015). Perfil de pessoas idosas vivendo com HIV/aids em Pelotas, sul do Brasil, 1998 a 2013. Epidemiol Serv Saúde, 24(1):79-86. Recuperado de http://www.scielo.br/scielo.php?script=sci_ arttext\&pid=S2237-

96222015000100079\&lng=en. http://dx.doi.org/10.5123/S167949742015000100009.

Alencas, R.A., Ciosak, S.I. (2014). O diagnóstico tardio e as vulnerabilidades dos idosos vivendo com HIV/aids. Rev EsC Enferm USP, 49(2):229-35.

Almeida, E.L., Araújo, G.B.S., Santos, V.A., Bustorff, L.A.C.V., Pereira, A.V.L., Dias, M.D. (2011). Adesão dos portadores do HIV/AIDS ao tratamento: fatores intervenientes. Rev Min Enferm, 15(2): 20816.

Bezerra, V.P., Serra, M.A.P., Cabral, I.P.P., Moreira, M.A.S.P., Almeida, S.A., Patrício, A.C.F.A. (2015). Práticas preventivas de idosos e a vulnerabilidade ao HIV. Rev
Gaúcha Enferm, 36(4):70-6. Recuperado de http://www.scielo.br/scielo.php?script=sci_ arttext\&pid=S198314472015000400070\&lng=en. http://dx.doi.org/10.1590/1983$\underline{1447.2015 .04 .44787}$

Bittencourt, G.K.G.D., Moreira, M.A.S.P., Meira, L.C.S., Nóbrega, M.M.L., Nogueira, J.A., Silva, A.O. (2015). Concepções de idosos sobre vulnerabilidade ao HIV/Aids para construção de diagnósticos de enfermagem. Rev Bras Enferm, 68(4):57985.

Burigo, G.F., Fachini, I.H., Garetti, B., Streicher, C.C.I., Rosa, R.S. (2015). Sexualidade e comportamento de idosos vulneráveis a doenças sexualmente transmissíveis. CuidArte Enferm., 9(2):14853.

Dornelas, N.J., Nakamura, A.S., Cortez, L.E.R., Yamaguchi, M.U. (2015). Doenças sexualmente transmissíveis em idosos: uma revisão sistemática. Ciênc saúde coletiva, 20(12):3853-3864. Recuperado de http://www.scielo.br/scielo.php?script=sci_ arttext\&pid=S141381232015001203853\&lng=en. http://dx.doi.org/10.1590/1413$\underline{812320152012.17602014}$

Driemeier, M., Andrade, S.M.O., Pontes, E.R.J.C., Paniago, A.M.M., Cunha, R.V. (2012). Vulnerability to AIDS among the elderly in an urban center in central Brazil. Clinics, 67(1):19-25. Recuperado de http://www.scielo.br/scielo.php?script=sci_ arttext\&pid=S1807$59322012000100004 \& \operatorname{lng}=$ en. http://dx.doi.org/10.6061/clinics/2012(01)0 $\underline{4}$

Duarte, M.T.C., Parada, C.M.G.L., Souza, L.R. (2014). Vulnerabilidade de mulheres vivendo com HIV/Aids. Rev Latino-Am Enferm, 22(1):1-8. Recuperado de 
Revista científica de la Asociación de Historia y Antropología de los Cuidados (Universidad de Alicante)

http://www.scielo.br/pdf/rlae/v22n1/pt_010 4-1169-rlae-22-01-00068.pdf

Figueiredo, L.G., Silva, R.A.R., Silva, I.T.S., Souza, K.G.S., Silva, F.F.A. (2013). Percepção de mulheres casadas sobre o risco de infecção pelo HIV e o comportamento preventivo. Rev enferm UERJ, 21(esp.2):805-11. Recuperado de http://www.facenf.uerj.br/v21esp2/v21e2a1 8.pdf .

Garcia, G., Lima, L.F., Silva, J.B., Andrade, L.D.F., Abrão, F.M.S. (2012). Vulnerabilidade dos Idosos frente ao HIV/Aids: Tendências da Produção Científica Atual no Brasil. DST- $J$ bras Doenças Sex Transm, 24(3):183-8, 2012.

Lazarotto, A.R., Santos, V.S., Reichert, M.T., Quevedo, D.M., Fossatti, P., Santos, G.A. et al. (2013). Oficinas educativas sobre HIV/Aids: uma proposta de intervenção para idosos. Rev Bras Geriatr Gerontol, 16(4):833-43, 2013.

Lima, T.C., Freitas, M.I.P. (2012). Caracterização de população com 50 anos ou mais atendida em serviço de referência em HIV/Aids, Brasil. Rev Ciênc Méd, 22(2):7786.

Mendes, K.D.S., Silveira, R.C.C.P., Galvão, C.M. (2008). Revisão integrativa: método de pesquisa para a incorporação de evidências na saúde e na enfermagem. Texto contexto enferm, 17(4):758-64. Recuperado de http://www.scielo.br/scielo.php?script=sci_ arttext\&pid=S010407072008000400018\&lng=en. http://dx.doi.org/10.1590/S0104$\underline{07072008000400018 .}$.

Mpondo, B.C.T. (2016). HIV Infection in the Elderly: Arising challenges. J Aging Res, 2016(1): 20-37. Recuperado de https://www.ncbi.nlm.nih.gov/pmc/articles/ $\underline{\text { PMC4993911/ }}$

Okuno, M.F.P., Gomes, A.C., Meazzini, L., Scherrer, J.G., Belasco, J.D., Belasco, A.G.S. (2014). Qualidade de vida de pacientes idosos vivendo com HIV/AIDS. Cad Saúde Pública, 30(7):1551-9. Recuperado de http://www.scielo.br/scielo.php?script=sci_ arttext\&pid=S0102-

311X2014000701551\&lng=en. http://dx.doi.org/10.1590/0102311X00095613.

Paz, M.A., Alencar, J.M.N., Sousa, C.L.A., Anjos, U.U., Nogueira, J.A., Rodrigues, J.A. (2013). A influência do uso da camisinha masculina por idosos na vulnerabilidade ao HIV: Uma revisão sistemática com metaanálise. DST- J bras Doenças Sex Transm, 25(3):150-6.

Quadros, K.A.N., Campos, C.R., Soares, T.E., Silva, F.M.R. (2016). Perfil epidemiológico de idosos portadores de HIV/AIDS atendidos no serviço de emergência especializada. RECOM, 6(2):2140-46.

Rodrigues, N.C.P., Almeida, A.S., Braga, J.U., O'Dwyer, G., Apratto, J.P.C., Daumas, R.P. et al. (2015). Spatial dynamics of AIDS incidence in the elderly in Rio de Janeiro, Brazil, 1997-2011. Cad Saúde Pública, 31(8):1721-31. Recuperado de http://www.scielo.br/scielo.php?script=sci_ arttext\&pid=S0102311X2015000801721\&lng=en. http://dx.doi.org/10.1590/0102311X00152914

Santana, P.P.C., Andrade, M., Santos, E.I., Espírito Santo, F.H., Braga, A.L.S., Teixeira, P.A. (2015). Evidências científicas de enfermagem acerca do HIV/AIDS entre 
idosos: Uma revisão integrativa de literatura. Rev Baiana Enferm, 29(3):278-89.

Santos, A.F.M., Assis, M. (2011). Vulnerabilidade das idosas ao HIV/AIDS: despertar das políticas públicas e profissionais de saúde no contexto da atenção integral: revisão de literatura. Rev Bras Geriatr Gerontol, 14(1):147-57.

Silva, L.C., Felício, E.E.A.A., Cassétte, J.B., Soares, L.A., Morais, R.A., Prado, T.S. et al. (2015). Impacto psicossocial do diagnóstico de HIV/aids em idosos atendidos em um serviço público de saúde. Rev bras geriatr gerontol, 18(4):821-33. Recuperado de http://www.scielo.br/scielo.php?script=sci_ arttext\&pid=S1809-

98232015000400821\&lng=en.

http://dx.doi.org/10.1590/1809-

9823.2015.14156.

Silva, M.M., Vasconcelos, A.L.R., Ribeiro, L.K.N.P. (2013). Caracterização epidemiológica dos casos de AIDS em pessoas com 60 anos ou mais, Pernambuco, Brasil, 1998 a 2008. Cad Saúde Pública, 29(10):2131-5. Recuperado de http://www.scielo.br/scielo.php?script=sci_ arttext\&pid=S0102-

$311 X 2013001000028 \& \operatorname{lng}=$ en.

http://dx.doi.org/10.1590/0102-

$\underline{311 X 00161112}$

Souza, L.P.S., Oliveira, M.V.R., Silveira, W.R.M., Figueiredo, M.F.S., Messias, R.B., Silva, J.R. (2012). Análise da clientela idosa portadora de HIV atendida em um centro ambulatorial em Montes Claros, Minas Gerais. Rev bras geriatr gerontol, 15(4): 767-76. Recuperado de http://www.scielo.br/scielo.php?script=sci_ arttext\&pid=S1809-

98232012000400015\&lng=en.

http://dx.doi.org/10.1590/S1809-

98232012000400015
Ultramari, L., Moretto, P.B., Gir, E., Canini, S.R.M.S., Teles, A.S., Gaspar, J., Machado, A.A. (2011). Perfil clínico e epidemiológico da infecção pelo HIV/aids em idosos. Rev Eletr Enf, 13(3)405-12. Recuperado de https://www.revistas.ufg.br/fen/article/view /11816/10641

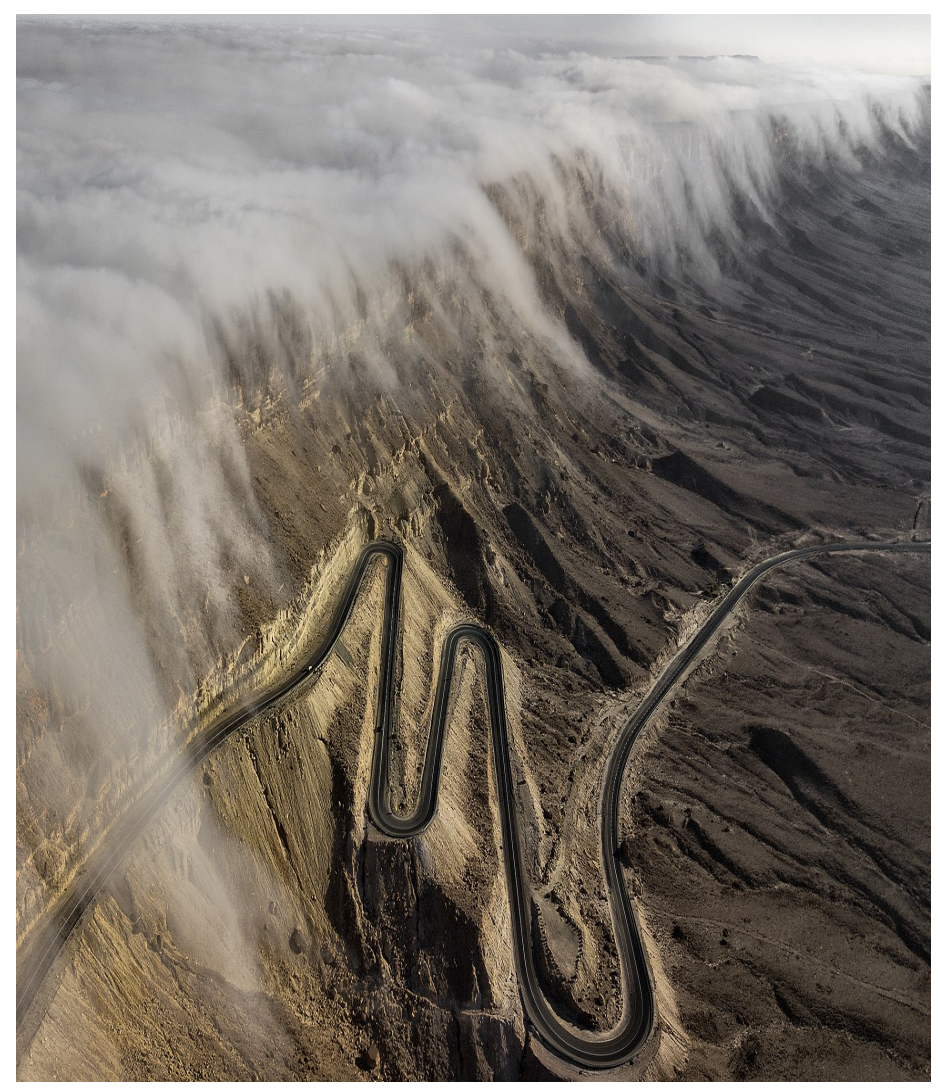

Fuente: https://commons.wikimedia.org/wiki/ Main_Page\#/media/File:-_מכתש רמון _גלישת_עננים (cropped).jpg 